\title{
Health Education in the Application of Improved Breathing Exercises on Patients with Chronic Obstructive Pulmonary Ventilation
}

\author{
Shuqin Song, Jingchun An, Xiuli Chen
}

The Third Affiliated Hospital of Qiqihar Medical College, Qiqihar, Heilongjiang, 161000

Keywords: improved breathing exercises; chronic obstructive pulmonary disease; health education

\begin{abstract}
The integration of health education into improved breathing exercises in the care of patients with chronic obstructive pulmonary ventilation can effectively improve patient compliance, help patients to actively participate in, actively cooperate with the diagnosis and treatment and nursing, cooperate with health education, and increase the improvement of breathing exercises. The effect promotes its functional recovery and psychological recovery. This article systematically introduces the purpose, principles, health education timing, and health education content of health education for patients with chronic obstructive pulmonary mechanical ventilation with improved breathing exercises.
\end{abstract}

\section{Introduction}

Chronic Obstructive Pulmonary Disease (COPD), referred to as COPD, is a common group of diseases that can be prevented and treated, characterized by persistent airflow limitation, and the patient's prevalence and mortality are both high. In recent years, 20245 adults have been surveyed in 7 regions in China. The prevalence of COPD in the population over 40 years old in China is $8.2 \%$. Chronic obstructive pulmonary disease is an incomplete reversible airflow limitation that is progressively associated with an abnormal inflammatory response to harmful gases or particulates such as lung inhalation of tobacco smoke. It is a common and frequently-occurring disease with high prevalence and mortality. According to the research of the World Health Organization, COPD has caused a huge social and family economic burden, and by 2020 will become the fifth largest economic burden on the world's disease. After mechanical ventilation, the patient had the most difficulty in weaning and had the longest average weaning time. Therefore, effective lung rehabilitation training should be carried out step by step, which can effectively improve the quality of life of patients. Through this study, improved respiratory training for patients with COPD has increased awareness of active training, increased limb muscle function and exercise capacity, enhanced patient confidence, prevention of ventilator dependence, physical and psychological preparation for offline, extended The intermittent off-line time is of positive significance in improving the success rate of offline and shortening the number of days of mechanical ventilation, etc., and is crucial to the health education of patients in the application of improved breathing exercises.

\section{Clinical data and improved respiratory care methods}

Clinical data Between March 2015 and March 2017, 80 patients who met the COPD diagnostic criteria in the respiratory department of the Third Affiliated Hospital of Qiqihar Medical College were selected as study subjects. According to different nursing methods, it was divided into observation group and control group. Inclusion criteria: mechanical ventilation time $\geq 72$ hours; no limb movement disorders; sober, cooperative; stable vital signs. Exclusion criteria: merger of heart, brain, kidney, liver and other organs complications, malignant tumors; cerebrovascular sequelae, limb movement disorders. In the process of limb function training and improved respiratory function training, the effect of health education was observed.

Use modified breathing exercises. Including: limb function training, improved breathing function training, design table to record the patient's vital signs before and after each training, blood oxygen 
saturation, breathing machine parameters, training items, time, with or without discomfort. Physical function training: including: fist, flexion and extension of the elbow joint, double upper limbs lifting, lifting, ankle flexion and extension movement, double lower limb flexion $90^{\circ}$, straight leg elevation $30^{\circ}$, alternating legs, 2 times a day, morning and evening 1 time each. Modified Breathing Function Training: Mechanical Ventilation Patients who use spontaneous breathing patterns perform abdominal breathing and slow breathing exercises while performing physical function training. Abdominal breathing: the patient takes a seat or a semi-recumbent position, relaxes whole body, places one hand on the abdomen, puts one hand on the chest, expands the abdomen to the maximum when inhaling, and keeps the chest unmoved. When exhaling, maximize the The abdomen is shrunk, and the hand placed on the abdomen is slightly pressurized and the chest remains immobile. Slow breathing: breathing deep and slow, deep breathing $3 \sim 5 s$, breath 1 s, and then slowly exhale $3 \sim 5 s$, breath-hold 1s. 3 times a day, 1 time each morning, evening and evening, every 5 to 10 minutes, each interval time is not less than 4 hours. The training schedule is concentrated during the day and before 21:00 so as not to affect the patient's rest.

Clinical efficacy observation indicators. The rate of mechanical ventilation days, intermittent off-line, and intermittent offline success rate of two groups of patients were counted by the implementer, and the patient's heart rate, respiration, blood pressure, and transcutaneous oxygen saturation were recorded before and 10 minutes after each implementation of the modified breathing exercises.

\section{Implementation of health education}

Through health education, actively assist patients and their families in adopting behaviors and lifestyles that are conducive to health, eliminate or reduce adverse factors that affect patients' rehabilitation, improve self-care and self-care capabilities, prevent ventilator dependence, and do a good job of off-line Physiological and psychological preparations to reduce the occurrence of complications, to achieve the overall quality of prevention, rehabilitation, health and other improvements.

Incorporating health education into improved breathing exercises In the application of chronic obstructive pulmonary ventilation to patients, increase the understanding of the medical principles of nursing work by patients and their families, improve patient compliance, provide quality patient care services, and improve patients Satisfaction.

Prior to the application of the ventilator, prior to the application of the ventilator, the patient and family members were given a health education using the time of the patient's visit.

In the case of ventilator applications, the ventilator is used for the entire process of the patient's health education after $1 \mathrm{~d}$ of use, and before the off-line application of improved breathing exercises in the pre- and during-process health education.

Take full advantage of any opportunity to contact the patient for health education: In the morning and evening care, medication, patrolling wards, and nursing care monitoring in real time to the patient's health education.

Personalized Education Principles Health education should vary from person to person and be tailored to the patient's age, gender, education level, occupation, personality, etc., and combined with the patient's condition needs, to give personalized health guidance. For example, the emphasis on the education of patients undergoing initial and multiple inpatient visits is different; the way of education for patients of different cultures and professions is different; the education forms of patients with different ages of the same disease are different.

Appropriate and timely education principles Health education should choose the right time and do proper health education. The contents of pre-operative ventilator and nursing and application education should be different. It is more appropriate to choose the timing for performing improved breathing exercises before going offline.

The principle of combining encouragement and guidance, appropriate repetitive education in the process of health education, pay attention to encouragement of patients, establish a solid support system with the family members, and establish the confidence of the patient in overcoming the 
disease; The important content and the elderly should pay attention to repeat education, and one content should not be too much.

Principle of joint participation The health education process is a process in which medical care, patients, and family members participate in the process. Through joint participation, patients and their families can actively cooperate with the medical staff's diagnosis and treatment and nursing work to improve their ability to take care of themselves and prevent related risks. .

Health education affects the behavior of individuals and groups through the dissemination of disease health knowledge and self-care technology, thereby eliminating the risk factors that may cause the disease and achieving the purpose of preventing disease and promoting health. In recent years, with the changes in people's living habits, the incidence of many diseases that are closely linked to people's bad habits has become higher. Drug treatment is an important method of disease treatment. It is also important to change the patient's bad health behaviors and habits through health education.

Ventilation of Ventilator Before Treatment: Introduce ward environment, hospitalization instructions, etc.; introduce the understanding of medical staff and patients in the same room to reduce the tension of patients; introduce the purpose, methods, etc. of related laboratory tests; conduct hospital assessment to understand the patient's disease Knowledge level and health education needs; to explain to patients and their families the condition and possible outcomes of the disease, explain the work, to understand the purpose, methods and importance of non-invasive ventilation.

During Ventilator Ventilation Treatment and Modified Breathing Exercises: Select a suitable mask, properly fix the headband, maintain a uniform tension, and use the wound protection patch prone to face pressure ulcers; instruct the patient to take a proper position and ensure The airway is open. Guide patients to inhale, mouth breath, avoid mouth breathing, reduce flatulence, cause man-machine confrontation; for the first time in patients treated with a ventilator, the medical staff waited for an hour at the bedside and adjusted the parameters of the ventilator at any time according to the patient's condition. Reduce the patient's discomfort; Instruct the patient to intermittently drink 1500-2000ml daily, teach him an effective method of coughing, and encourage his own cough. For those who can't cough on their own, they should turn over and shoot back, mechanically suck, and the nurse regularly checks the temperature of the humidifier and The amount of water to ensure a good moisturizing effect; guide the patient through the action demonstration of lung function rehabilitation exercise method; guide patients with effective coughing and correct shoot back, phlegm and other methods to maintain airway patency; introduce the pharmacological effects of the drug used, precautions and so on.

Dietary care Health education instructs patients to eat high-protein, high-calorie, light-digestible foods, avoid eating cold, spicy, greasy foods; reduce carbohydrate intake, avoid excessive carbon dioxide, increase ventilation load; guide patients How to carry out language exchanges in the process of carrying out the machine and complete basic life needs.

Health education for psychological care. After the patient is admitted to the hospital, the nursing staff needs to fully understand the patient's general conditions, such as age, gender, actual condition, and other relevant aspects. At the same time, the patient is evaluated and analyzed accordingly, and a targeted nursing plan is formulated according to the assessment of the patient. Nursing staff need to inform patients of the causes, treatment methods and rehabilitation knowledge of COPD, help patients to raise awareness of the disease, and inform patients of the impact of smoking on the disease, and eliminate negative emotions through psychological care.

Physical Function Training Health Education. Including: fists, flexion and extension of the elbow joint, double upper limbs lifting, lifting, ankle flexion and extension movement, double lower limb flexion $90^{\circ}$, straight leg raising $30^{\circ}$, alternating legs, 2 times a day, morning and evening.

Improve breathing function training Health education. Mechanical ventilation uses patients with spontaneous breathing patterns and abdominal training and slow breathing exercises while performing physical function training. Abdominal breathing: the patient takes a seat or a semi-recumbent position, relaxes whole body, places one hand on the abdomen, puts one hand on 
the chest, expands the abdomen to the maximum when inhaling, and keeps the chest unmoved. When exhaling, maximize the The abdomen is shrunk, and the hand placed on the abdomen is slightly pressurized and the chest remains immobile. Slow breathing: breathing deep and slow, deep breathing $3 \sim 5$ s, breath 1s, and then slowly exhale $3 \sim 5$ s, breath-hold $1 \mathrm{~s}$. 3 times a day, 1 time each morning, evening and evening, every 5 to 10 minutes, each interval time is not less than 4 hours.

\section{Discussion}

Improved respiratory function training patients with improved respiratory function. Patients with COPD need to increase their respiratory rate to compensate for dyspnea. Most of them rely on chest-assisted breathing with assisted respiratory muscles. The patients are prone to respiratory muscle fatigue, resulting in short intermittent offline or offline failure. Abdominal breathing can increase the range of motion of the diaphragm. Every $1 \mathrm{~cm}$ of movement of the diaphragm can increase the ventilation volume of the lung by 250 to $300 \mathrm{ml}$, which significantly increases the effective ventilation volume and lung flexibility. Slow breathing allows the patient to consciously slow down the respiratory rate, reduce ineffective ventilation caused by shallow quick breathing, and avoid respiratory muscle fatigue. Improved respiratory function training during mechanical ventilation in COPD patients helps patients change their breathing patterns, improve oxygenation, and improve their respiratory function.

Increased awareness of active patient training. Prepare Physiological and Psychological Preparation for Offline COPD Patients with mild gas obstruction may experience weakened limb muscle function. Some patients with mechanical ventilation use analgesic sedatives earlier to relieve pain, avoid increased oxygen consumption, and reduce patients' mobility. .

Health education improved the confidence of patients with improved respiratory function training, changed behavioral patterns, improved adherence to treatment care, increased self-care awareness, increased the effect of improved breathing exercises, and promoted functional recovery and psychological rehabilitation.

Due to the large number of patients with COPD in this region, the high incidence of COPD patients in spring and autumn, mechanical ventilation, the phenomenon of prolonged mechanical ventilation days, and intermittent offline success rate, through this study, can shorten the number of mechanical ventilation days, extend Intermittent offline time, improve intermittent offline success rate. And can reduce the number of patients breathing, improve skin oxygen saturation, is conducive to improving the patient's breathing, oxygenation.

\section{Acknowledgements}

Fund Project: Social Development Project of Science and Technology Bureau of Qiqihar City (RKXZD-2016008)

\section{References}

[1] Zhang Xiaonv. The role of physical function exercise in patients with chronic obstructive pulmonary disease and mechanical ventilation [J]. Chinese Journal of Practical Nursing, 2006, 22 (2) :9-10.

[2] Shen Manling. Effects of respiratory function training on patients with chronic obstructive pulmonary disease during remission[J]. Journal of Nursing, 2007, 14(5): 54-55.

[3] He Shengnan, Niu Meixi, Ni Jingyu, et al. Health education path in the stable period of chronic obstructive pulmonary disease in inpatient health education application [J]. Nursing Research, 2015, 5(5): 552-555. 